\title{
Erratum to: Bacillus thaonhiensis sp. nov., a New Species, was Isolated from the Forest Soil of Kyonggi University by Using a Modified Culture Method
}

\author{
H. T. Van Pham · Jaisoo Kim
}

Published online: 14 June 2014

(C) Springer Science+Business Media New York 2014

Erratum to: Curr Microbiol (2014) 68:88-95

DOI 10.1007/s00284-013-0443-1

The original version of this article unfortunately contained a mistake. The etymology of species name was incorrect based on The International Code of Nomenclature of Prokaryotes. The correct epithet is thaonhiae (tha.o.nhi'ae, N.L. gen. fem. n. thaonhiae ...) as given here.

The online version of the original article can be found under doi: 10 . 1007/s00284-013-0443-1.

H. T. Van Pham · J. Kim ( $\square)$

Department of Life Science, Graduate School of Kyonggi

University, Suwon, Gyeonggi-Do 443-760, South Korea

e-mail: jkimtamu@kyonggi.ac.kr; jkimtamu@kgu.ac.kr 